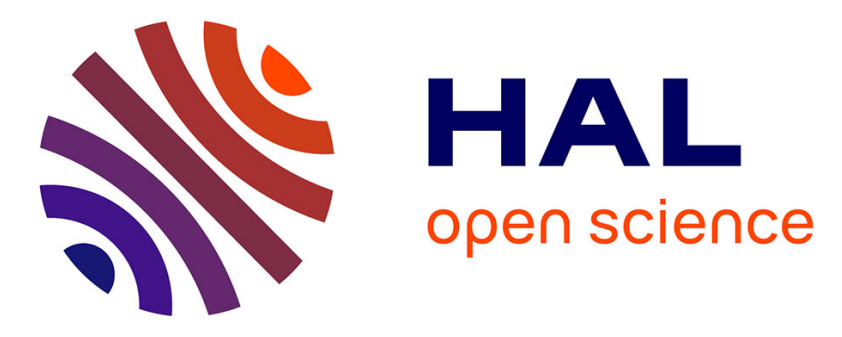

\title{
Tailoring and visualising pore openings in gelatin-based hydrogel foams
}

Friederike Dehli, Alexander Southan, Wiebke Drenckhan-Andreatta, Cosima Stubenrauch

\section{- To cite this version:}

Friederike Dehli, Alexander Southan, Wiebke Drenckhan-Andreatta, Cosima Stubenrauch. Tailoring and visualising pore openings in gelatin-based hydrogel foams. Journal of Colloid and Interface Science, 2021, 588, pp.326-335. 10.1016/j.jcis.2020.12.064 . hal-03410658

\section{HAL Id: hal-03410658 \\ https://hal.science/hal-03410658}

Submitted on 4 Nov 2021

HAL is a multi-disciplinary open access archive for the deposit and dissemination of scientific research documents, whether they are published or not. The documents may come from teaching and research institutions in France or abroad, or from public or private research centers.
L'archive ouverte pluridisciplinaire HAL, est destinée au dépôt et à la diffusion de documents scientifiques de niveau recherche, publiés ou non, émanant des établissements d'enseignement et de recherche français ou étrangers, des laboratoires publics ou privés. 


\title{
Tailoring and visualising pore openings in gelatin-based hydrogel foams
}

\author{
Friederike Dehli ${ }^{1}$, Alexander Southan ${ }^{2}$, Wiebke Drenckhan ${ }^{3}$, Cosima Stubenrauch ${ }^{1,4^{*}}$ \\ ${ }^{1}$ Institute of Physical Chemistry, University of Stuttgart, \\ Pfaffenwaldring 55, 70569 Stuttgart, Germany \\ ${ }^{2}$ Institute of Interfacial Process Engineering and Plasma Technology, \\ University of Stuttgart, Nobelstraße 12, 70569 Stuttgart, Germany \\ ${ }^{3}$ Institut Charles Sadron, 23 rue de Loess, Strasbourg, 67034, France \\ ${ }^{4}$ Institute of Advanced Studies (USIAS), University of Strasbourg, F-67000 France \\ *Corresponding Author: cosima.stubenrauch@ipc.uni-stuttgart.de, 0049711 685-64470
}

\begin{abstract}
Hypothesis: While tailoring the pore diameters in hydrogel foams has been demonstrated in numerous studies, fine control over the diameters of the pore openings is still a challenge. We hypothesise that this can be achieved by controlling the size of the thin films which separate the bubbles in the liquid foam template. If this is the case, systematic changes of the template's gas fraction $\phi$ (the higher $\phi$, the larger are the thin films) will lead to corresponding changes of the pore opening diameter.

Experiments: Since the size of the thin films depends on both bubble size $\left\langle D_{\mathrm{b}}\right\rangle$ and gas fraction $\phi$, we need to decouple both parameters to control the film size. Thus, we generated foams with constant bubble sizes via microfluidics and adjusted the gas fractions via two different techniques. The foams were solidified using UV light. Subsequently, they were analysed with confocal fluorescence microscopy.

Findings: We were able to change the pore opening diameter $\left\langle d_{\mathrm{p}}\right\rangle$ at a constant pore diameter $\left\langle D_{\mathrm{p}}\right\rangle$ by adjusting the gas fraction of the foam template. The obtained $\left\langle d_{\mathrm{p}}\right\rangle /\left\langle D_{\mathrm{p}}\right\rangle$ ratios are between those obtained theoretically for disordered foams and FCC ordered foams, respectively.
\end{abstract}

\section{Keywords}

foam templating, gelatin methacryloyl, microfluidics, pore opening, biopolymer scaffolds 


\section{Introduction}

Hydrogel foams based on biopolymers such as alginate, hyaluronic acid or gelatin have gained increasing attention in the field of biomaterials science because they combine the porous foam structure with the unique material properties of a hydrogel. Hydrogels are three-dimensionally cross-linked hydrophilic polymer networks that swell to equilibrium in aqueous media. ${ }^{1,2}$ Due to their high water content ${ }^{3}$, as well as their biocompatibility and biodegradability ${ }^{4-6}$, hydrogels based on biopolymers are studied extensively as functional materials in various fields, e.g. tissue engineering or drug delivery. ${ }^{7-10}$ What is of particular interest in this context is the biopolymer gelatin, which contains amino acid sequences relevant for cell adhesion as well as cleavage sites for biodegradation. ${ }^{11,12}$ However, the diffusion of small molecules such as drugs or nutrients through hydrogels is often hindered by the small mesh sizes (up to tens of nanometres) of the material. ${ }^{13-15}$ Thus, the generation of hydrogel foams with pore diameters ranging from tens to hundreds of micrometres has been investigated thoroughly.

One suitable method for the generation of hydrogel foams based on biopolymers is liquid foam templating. ${ }^{16-19}$ With the help of this technique, a liquid foam is generated from an aqueous polymer solution and is subsequently solidified by cross-linking of the polymer. As the morphology of the hydrogel foam is determined by the structure of the liquid template, the key parameters of the material, i.e. the pore diameter and the pore opening diameter, can be finetuned by tailoring the liquid template. Both pore diameter and pore opening diameter play a key role for the material properties of polymeric foams. ${ }^{20,21}$ In the field of tissue engineering, relations between the differentiation and colonization behaviour of cells on the one hand and the pore diameter as well as the pore opening diameter on the other hand have been demonstrated in numerous studies. ${ }^{22-25}$ To tailor the structure of the liquid template and thus the morphology of the hydrogel foam, microfluidic foaming has been examined extensively. ${ }^{16,18,21,26}$ With the help of this technique, a gas and a liquid phase are pushed through a chip with a micrometric constriction, which results in the pinch-off of bubbles. As this pinch- 
off occurs periodically, the generated bubbles have the same diameter, i.e. they are monodisperse. The bubble diameter of the liquid template and thus the pore diameter of the hydrogel foam can be conveniently tailored by varying the gas pressure and/or the liquid flow rate inside the microfluidic chip. This has been demonstrated for hydrogel foams based on chitosan, alginate and gelatin. ${ }^{16,18,21,26}$ The bubble diameter is further determined by the size of the constriction. While this technique focuses on the variation of the pore diameter, the variation of the pore opening diameter has received little attention up until now. The mechanism of the pore opening process is still not very well understood. However, it can be assumed that the contact of two neighbouring bubbles leads to the formation of a contact zone between them. As these contact zones are subject to capillary forces generated by the surrounding struts, the thickness of the contact zone is reduced to a surfactant double layer. During cross-linking and freeze-drying, these films break and pore openings are generated (see Figure 1).

1)

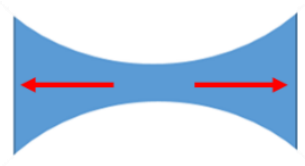

2)

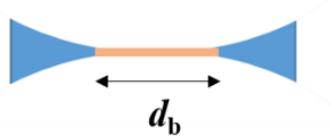

3)

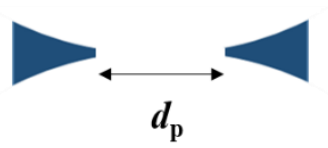

Figure 1: Proposed mechanism for the generation of pore openings. (1) Drainage of the polymer solution (blue) between two neighbouring bubbles due to capillary forces (red arrows). (2) Expulsion of the polymer from the film generating a thin film with diameter $d_{\mathrm{b}}$. (3) Upon solidification of the polymer (dark blue) the thin film ruptures and a pore opening with diameter $d_{\mathrm{p}}$ is generated.

Generally speaking, in liquid foam templating, the pore opening diameter correlates, to a first approximation, directly with the diameter of the thin liquid film which separates neighbouring bubbles. For foams with a high gas fraction $\phi(>90 \%)$ one can show by means of a simple scaling argument that the diameter $\left\langle d_{\mathrm{p}}\right\rangle$ of the pore opening is related to the pore diameter $\left\langle D_{\mathrm{p}}\right\rangle$. It holds

$$
\left\langle d_{\mathrm{p}}\right\rangle \sim(1-\sqrt{1-\Phi})\left\langle D_{\mathrm{p}}\right\rangle
$$


For lower gas fractions, this relationship becomes a bit more complicated, as will be discussed in Section 3.3. Nevertheless, it shows the importance of decoupling the gas fraction and the pore size for explicit pore opening control. This was shown qualitatively for polyurethane foams by Testouri et al. ${ }^{27} \mathrm{~A}$ more systematic approach was developed for geopolymer- or gelatinbased foams that were generated from a foamed precursor solution which was then mixed with the geopolymer- or gelatin-containing dispersion / solution. ${ }^{21,28,29}$ However, this approach is time-consuming, requires multiple formulations, and has high demands on foam stability. These requirements cannot always be met when using photo cross-linkable hydrogel formulations. The decoupling of pore size and gas fraction can also be achieved by varying the surfactant concentration, which was examined for systems based on alginate and methacrylated dextran. ${ }^{18,30}$ Despite the fact that this is a very elegant concept, high surfactant concentrations are required, which limits potential applications.

In the present study, we used microfluidics for the generation of monodisperse liquid foams with a specific bubble diameter. The continuous phase of the foams consisted of an aqueous solution of gelatin methacryloyl. In order to tailor the pore opening diameter, we systematically adjusted the gas fraction of the foams independently of the bubble size using two different methods. In the first method, the polymer solution was added to the liquid foam template made at high gas fraction from the very same polymer solution. In the second method, the bubbling frequency during foam generation was tailored by varying the liquid flow rate and gas pressure while keeping the ratio of these parameters constant. This effect was examined by Garstecki et $a l .{ }^{31}$ for an aqueous surfactant solution, but has never been used to tailor the pore opening diameters in polymer foams. Another question we want to address is how hydrogel foams can be characterised. Typically, hydrogel foams are characterized by scanning electron microscopy, $\mu$-CT or bright field microscopy. The downside of these methods is that they are expensive and time-consuming or do not adequately visualise the equilibrium state, i.e. the swollen state of the material. Here, we use confocal fluorescence microscopy to characterise the material in the 
swollen state. The paper is organised as follows: Firstly, we briefly report and discuss the use of confocal fluorescence microscopy for the characterisation of gelatin-based hydrogel foams. Secondly, we apply the two aforementioned approaches to decouple gas fraction and pore size. Finally, we compare the ratios of our experimentally determined pore opening diameters and pore diameters with predictions by Princen $^{32}$ and Arditty ${ }^{33}$ for disordered foams, as well as with predictions by Pitois et al. ${ }^{28,34}$ for foams with an FCC order.

\section{Experimental}

\subsection{Chemicals}

Gelatin (Type B, 232 bloom, batch \#635621) was purchased from Gelita (Germany). Methacrylic anhydride was purchased from Sigma Aldrich (Germany). Sodium 3trimethylsilylpropionate-2,2,3,3-d4 was purchased from Merck (Germany). Perfluorohexane (98\%, $85 \%$ n-isomer) was purchased from abcr (Germany). $\mathrm{D}_{2} \mathrm{O}$ was purchased from Deutero (Germany). Lithium phenyl-2,4,6-trimethylbenzoylphosphinate (LAP) was purchased from Carbosynth (UK). Plantacare 2000 UP was donated from Cognis (now BASF). Methylene blue was purchased from S3 chemicals (Germany). All chemicals were used as received. Deionised water was used for all experiments.

\subsection{Synthesis of gelatin methacryoyl}

Gelatin methacryloyl (GM) was prepared and analysed according to a procedure described by Claaßen et al..$^{35} 25.01 \mathrm{~g}$ of gelatin were dissolved in $250 \mathrm{~mL}$ of water at $40{ }^{\circ} \mathrm{C}$. After the gelatin was dissolved completely, the temperature was set to $37^{\circ} \mathrm{C}$. The $\mathrm{pH}$ was adjusted to 7.25 using a $4 \mathrm{M} \mathrm{NaOH}$ solution. $14.28 \mathrm{~mL}(89.5 \mathrm{mmol})$ of methacrylic anhydride were added dropwise. The amount of methacrylic anhydride corresponds to a 10 -fold molar excess with respect to free amino groups in gelatin $\left(0.35 \mathrm{mmol} \mathrm{g}^{-1}\right.$, according to van den Bulcke et al. $\left.{ }^{36}\right)$. 
Consequently, the resulting product is further denoted as GM10. The reaction mixture was stirred for $5 \mathrm{~h}$ while the $\mathrm{pH}$ was kept between 7.0 and 7.4 by addition of $4 \mathrm{M} \mathrm{NaOH}$ solution. The crude product was stored at $8{ }^{\circ} \mathrm{C}$ for two days, afterwards dialyzed for 5 days and freezedried using an Alpha 1 - 4 LSC lyophiliser from Christ (Germany). $18.2 \mathrm{~g}$ of GM10 were obtained, which corresponds to a yield of $73 \% .{ }^{1} \mathrm{H}-\mathrm{NMR}$ analysis in $\mathrm{D}_{2} \mathrm{O}$ was used to determine the degree of methacryloylation $(D M)$. TMSP was used as a standard. A $D M$ of $0.88 \mathrm{mmol} \mathrm{\textrm {g } ^ { - 1 }}$ was obtained.

\subsection{Generation of gelatin methacryloyl foams}

A solution of 20 wt. \% GM10, 0.14 wt. \% LAP and 0.1 wt. \% Plantacare 2000 UP was prepared with deionised water. The density of the solution was determined to be $1.056 \mathrm{~g} \mathrm{~cm}^{-3}$ by using a DMA 5000 M density meter from Anton Paar. All flasks were wrapped with aluminium foil and stored at $8{ }^{\circ} \mathrm{C}$ in the dark until further use to prevent early activation of the photo initiator. Liquid foams were produced using a polycarbonate chip produced by micromilling, with a constriction of $70 \mu \mathrm{m}$ in diameter (Figure 3). The flow of the gas phase was controlled by the gas pressure $p$. To this end, an OB1MK1 pressure controller from Elveflow was used, which was connected to a nitrogen tap. The pressure pump was also connected to a glass bottle containing a small amount of perfluorohexane. In this way, the gas phase contains traces of perfluorohexane, which hinders Ostwald ripening. The flow rate $v$ of the liquid phase was controlled with a Pump 11 Elite Syringe Pump from Harvard Apparatus. Bubbling in the microfluidic chip was monitored with a Nikon SMZ $745 \mathrm{~T}$ bright field microscope using a Mikrotron EoSensCL high speed camera. The accessible range of bubble diameters that can be produced using this microfluidic chip was assessed by varying the gas pressure $p$ and the liquid flow rate $v_{\mathrm{L}}$. The obtained data can be found in the Supporting Information (Figure S1). 


\subsubsection{Addition of polymer solution}

A liquid flow rate $v_{\mathrm{L}}$ of $7 \mu \mathrm{L} \mathrm{min} \mathrm{m}^{-1}$ and a gas pressure of 260 mbar were used to produce the foams. To analyse the bubble diameter of the liquid foam, pictures of foam monolayers taken with the Mikrotron EoSensCL high speed camera were analysed manually using the software ImageJ. In order to be able to add additional polymer solution to the resulting foams, a setup with connected and slanted outlet channels was used, where the same polymer solution that was used for the foaming was added with a defined flow rate $v_{\mathrm{A}}$. The liquid flow rate of the additional polymer solution is a direct control parameter for the liquid fraction of the generated foam. A scheme of the setup is depicted in Figure 2. As the additional polymer solution was added outside the microfluidic chip, undesirable feedback-loops on the bubble pinch-off at the constriction are prevented.

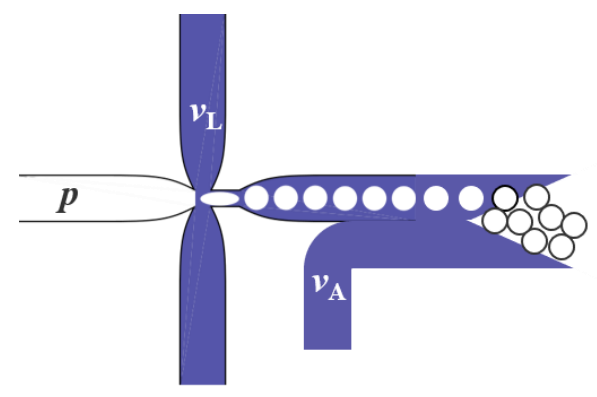

Figure 2: Scheme of experimental setup used for the generation of liquid foam templates. The bubble size is determined by the gas pressure $p$ and the liquid flow rate $v_{\mathrm{L}}$, whereas the gas fraction can be controlled by the liquid flow rate $v_{\mathrm{A}}$.

This setup has been used previously ${ }^{37}$ to add fast gelling cross-linking agents to liquid foams. The mixing of additional polymer solution with the foam is ensured by the moving bubbles. The flow rate $v_{\text {A }}$ was set by a second syringe pump (Pump 11 Elite, Harvard Apparatus) and was varied from $0 \mu \mathrm{L} \mathrm{min}{ }^{-1}$ to $11 \mu \mathrm{L} \mathrm{min}{ }^{-1}$. The resulting foams were collected in cylindrical PDMS moulds with a height of $2 \mathrm{~mm}$ and a diameter of $4 \mathrm{~mm}$. By choosing this small sample height, we aimed to minimise the influence of drainage on the liquid foams. During the collection of the foams, the samples were overflown with nitrogen to prevent oxygen inhibition 
during cross-linking. The foams were covered with a quartz glass and irradiated immediately with UV-A light for 4.5 min. An Omnicure Series 1500 UV lamp with a maximum emission spectrum between $360-370 \mathrm{~nm}$ was used. The UV light guide tip had a distance of $5 \mathrm{~cm}$ to the samples and was operated at $15 \%$ intensity. After cross-linking, the samples were frozen at $-60{ }^{\circ} \mathrm{C}$ for $30 \mathrm{~min}$ and freeze-dried. The dry samples were stored at room temperature until further use. The gas fraction $\phi$ of the foams was determined gravimetrically from the mass of the dried sample $m$, the sample volume in the liquid state $V_{1}$, the density $\rho$, and the concentration $c$ of the polymer solution, using equation (2).

$$
\phi=1-\frac{m}{c \cdot \rho \cdot V_{1}}
$$

All experiments were carried out three times. All errors correspond to standard deviations.

\subsubsection{Variation of the bubbling frequency}

Liquid foams were generated by varying the liquid flow rate $v_{\mathrm{L}}$ stepwisely from $20 \mu \mathrm{L} \mathrm{min}{ }^{-1}$ to $80 \mu \mathrm{L} \mathrm{min}{ }^{-1}$. The ratio of liquid flow rate to gas pressure was kept constant at $0.1 \mu \mathrm{L} \mathrm{min}{ }^{-1}$ $\mathrm{mbar}^{-1}$. As a result, the liquid fraction in the foam decreases with increasing liquid flow rate $v_{\mathrm{L}}$, while a constant bubble size is maintained. Pictures of bubbles produced in the microfluidic chip at different liquid flow rates and gas pressures are depicted in Figure 3.
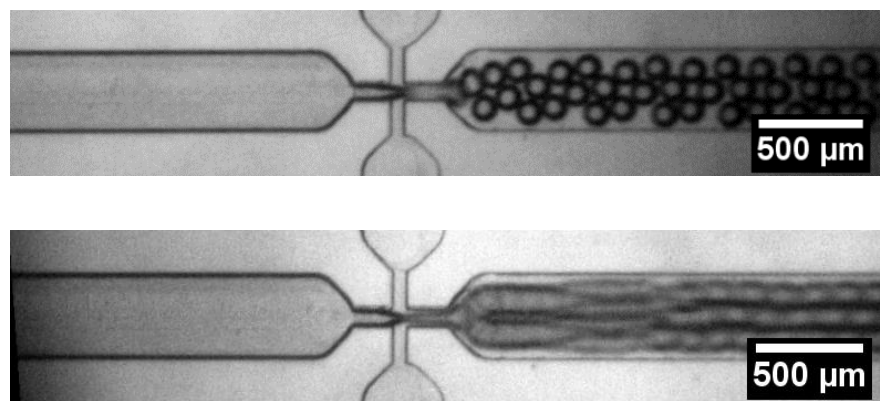

Figure 3: Generation of bubbles inside the microfluidic chip at different liquid flow rates and gas pressures: (top) $\nu_{\mathrm{L}}=20 \mu 1 \mathrm{~min}^{-1}, p=200 \mathrm{mbar}$; (bottom) $\nu_{\mathrm{L}}=70 \mu 1 \mathrm{~min}^{-1}, p=700 \mathrm{mbar}$. 
As the production parameters were changed for each sample, the bubble diameters were determined for each sample as described in Section 2.3.1. The samples were collected, crosslinked and freeze-dried as described in Section 2.3.1. The gas fraction of the samples was determined as described in Section 2.3.1. All experiments were carried out three times. All errors correspond to standard deviations.

\subsection{Staining and absorption spectroscopy}

As fluorescence microscopy relies on the excitation and emission of a fluorescing compound in the hydrogel matrix, a suitable dye which permanently stains the hydrogel had to be found. The isoelectric point of GM10 is 4.2, which leads to a negative net charge of the polymer in deionised water. ${ }^{38}$ In contrast to that, the dye methylene blue (Figure 4, left) is positively charged at a neutral $\mathrm{pH}$.<smiles>CN(C)c1ccc2c(c1)SC1CC(=[N+](C)[O-])C=CC1=N2</smiles>

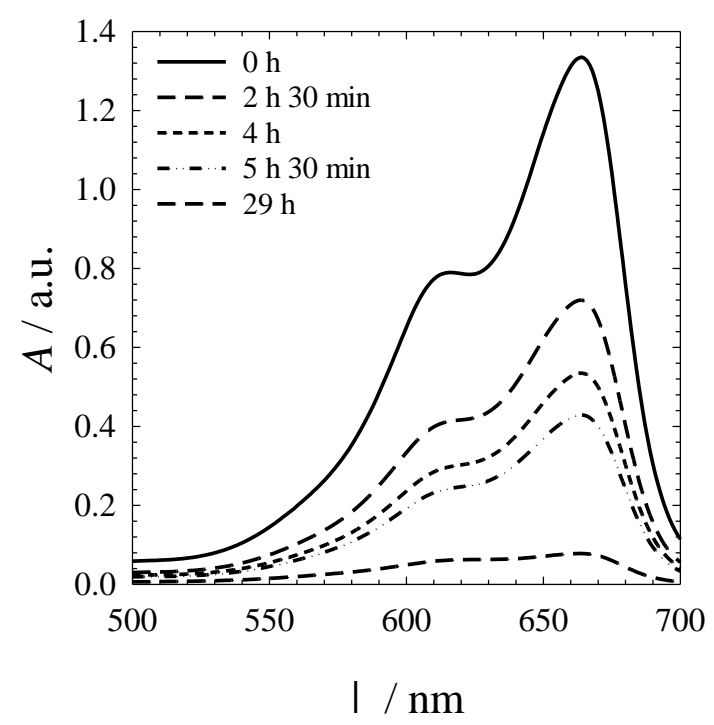

Figure 4: (left) Chemical structure of methylene blue. (right) Absorption spectra of a $5 \mathrm{mg} \mathrm{L}^{-1}$ aqueous methylene blue solution after different immersion times of a GM10 foam.

To stain samples with methylene blue, freeze-dried GM10 foams, which had a mass of about 2 $\mathrm{mg}$ and a volume of about $25 \mathrm{~mm}^{3}$, were immersed in $2 \mathrm{~g}$ of a $5 \mathrm{mg} \mathrm{L}^{-1}$ methylene blue solution under slight agitation (45 rpm) at room temperature. After $32 \mathrm{hrs}$ of staining, the methylene 
blue solution was removed, and the samples were immersed in $2 \mathrm{~g}$ water for at least 12 hours to remove unbound methylene blue from the gel phase as well as the pores. The samples were left at room temperature under slight agitation $(45 \mathrm{rpm})$ until characterization with fluorescence microscopy. To follow the complete adsorption of the dye in the foam, the progressive reduction of the signal of the supernatant in the visible range was measured with a Lambda 25 UV/VIS spectrometer from Perkin Elmer at different times. The VIS absorption spectra of the supernatant after different immersion times are shown in Figure 4 (right). After 29 hours of immersion, almost no methylene blue can be detected in the supernatant. No methylene blue visibly leaches out of the material. The absorption and emission maxima of methylene blue are located at $664 \mathrm{~nm}$ and $684 \mathrm{~nm}$, respectively. ${ }^{39}$

\subsection{Fluorescence microscopy}

An LSM 710 confocal laser scanning microscope from Zeiss (Germany) equipped with the Zen Black software was used to characterise the stained samples. 3D z-stacks composed of 2D pictures along the z-axis of the sample were recorded. To prevent the samples from drying during the measurement, they were placed onto a droplet of water. Consequently, the pores of the sample are filled with water. The excitation wavelength was $633 \mathrm{~nm}$. Emitted light was collected between 642 and $747 \mathrm{~nm}$. To be able to not only visualise the surface of the sample but the inside, the samples were cut. The cutting was carried out both horizontally and vertically to obtain cross-sections and longitudinal sections. Please note that the surface of the samples displays an ordered morphology as the bubbles crystallise under the confinement of the sample mold walls and the quartz glass plate. Exemplary pictures of the ordered surface areas for samples discussed in Section 3.2.1 and 3.2.2 are shown in the Supporting Information (Figure S2). As the crystallised areas are merely surface effects that are relevant for the first bubble layers only ${ }^{41}$, they are not considered for further analysis. 


\subsection{Picture analysis}

Compared to SEM, where the samples are cut at a random distance from the equatorial plane, stacks of $2 \mathrm{D}$ pictures can be recorded with confocal fluorescence microscopy. Thus, the acquired pore diameters were measured in pictures obtained close to the equatorial plane of the pores. Consequently, the maximum underestimation of the pore diameter depends on the distance of the $2 \mathrm{D}$ pictures in the stack, which is $9.6 \mu \mathrm{m}$ in our case. As a result, the maximum underestimation of the pore diameter in our study is only between $0.26 \mu \mathrm{m}$ and $0.33 \mu \mathrm{m}$. The obtained pictures were analysed using the software ImageJ (version 1.52p). A logarithmic transformation was applied to all pictures to account for skewed intensities due to light scattering when measuring deep inside the sample. Only $2 \mathrm{D}$ pictures of the $3 \mathrm{D}$ z-stack that captured either the pore diameter $\left\langle D_{\mathrm{p}}\right\rangle$ or the pore opening diameter $\left\langle d_{\mathrm{p}}\right\rangle$ in the focal plane were used (see Figure 5). At least 40 pore and pore opening diameters were analysed manually for each sample. Since all samples were prepared three times, we have a total of 120 values for each pore diameter and each pore opening diameter. The corresponding size distributions are shown in the Supporting Information (Figures S3-S6). When the pore opening diameters had slightly elliptical shapes in the $2 \mathrm{D}$ projections, the major axis was considered the pore opening diameter. All errors correspond to the standard deviation.
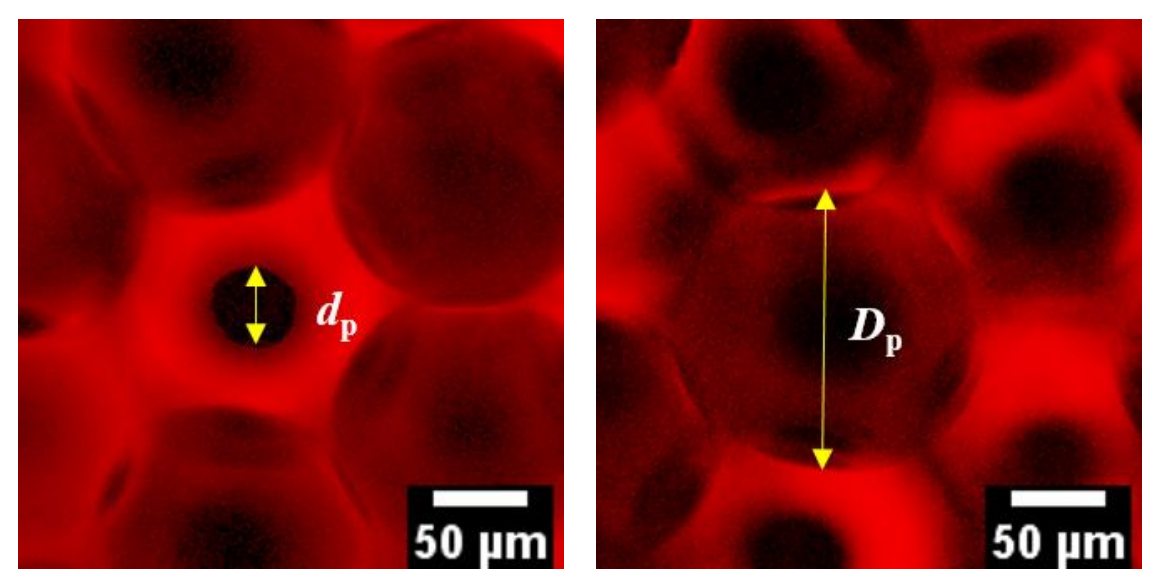

Figure 5: Determination of pore opening diameter (left) and pore diameter (right). Note that the $2 \mathrm{D}$ pictures refer to the same area in the sample, but are taken at different z-positions in the 3D stack to focus either on the pore opening or on the pore. 


\section{Results and Discussion}

\subsection{Visualisation of GM10 hydrogel foams}

One of the most important aspects to consider when tailoring the morphology of a material is a suitable characterization method. In this study, we want to demonstrate the possibility of using confocal fluorescence microscopy for the extensive characterization of GM10 hydrogel foams. A confocal fluorescence microscopy picture of a GM10 hydrogel foam stained with methylene blue which was immersed in water is shown in Figure 6 (right). As can be seen in the picture, it is only the hydrogel matrix that fluoresces (red areas in the picture) but not the water that fills the pores of the matrix (black areas in the picture).
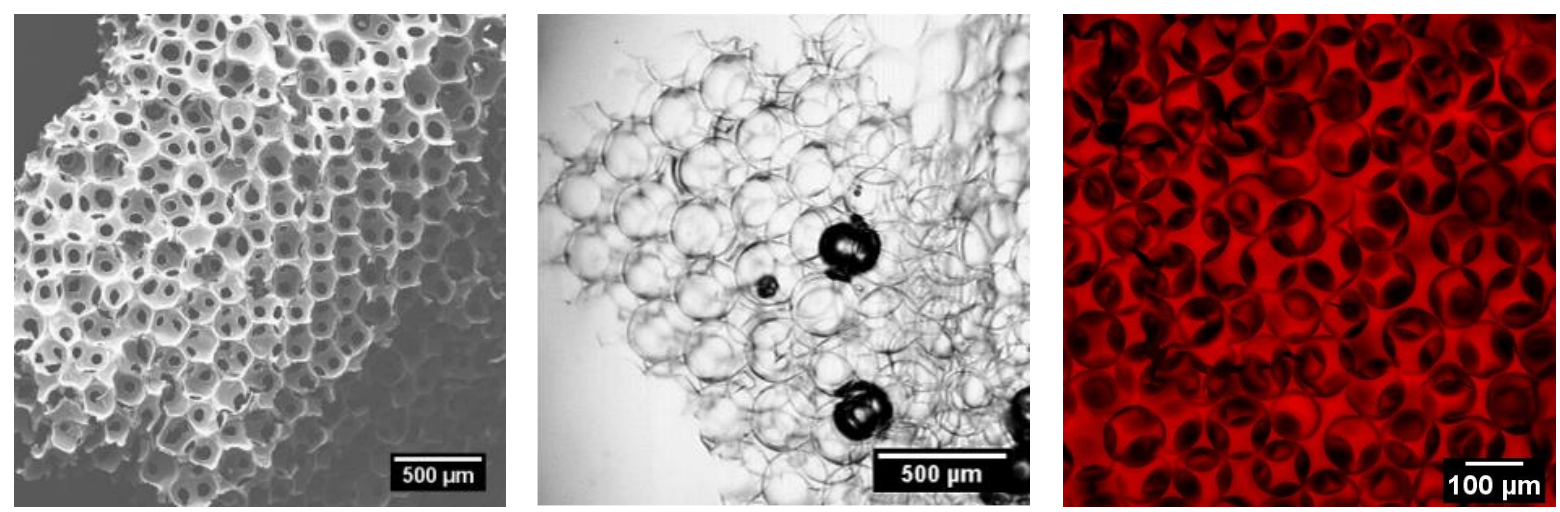

Figure 6: Pictures of a GM foam visualised with SEM (left) ${ }^{\mathrm{a}}$, bright field microscopy (middle) ${ }^{\mathrm{a}}$ and confocal fluorescence microscopy (right, maximum intensity projection).

${ }^{a}$ Adapted with permission from Dehli, F.; Rebers, L.; Stubenrauch, C.; Southan, A. Highly Ordered Gelatin Methacryloyl Hydrogel Foams with Tunable Pore Size, Biomacromolecules, 2019, 20, 2666 - 2674. Copyright 2019 American Chemical Society.

In a previous study, GM10 hydrogel foams were characterised using both SEM and bright field microscopy (Figure 6, left and middle). ${ }^{26}$ SEM has often been used as a standard characterization technique for hydrogel foams, ${ }^{16,24,40}$ as it visualises the interconnected structure and the pore diameter of the material (Figure 6, left). However, the material needs to be dried, which entails the formation of artefacts such as shrinkage. We have shown before that pore diameters in freeze-dried GM10 hydrogel foams are also smaller than pore diameters in GM10 foams swollen to equilibrium. ${ }^{26}$ What is yet unknown is the behaviour of pore openings during drying in general and the degree of pore opening shrinkage relative to the shrinkage of the pore 
diameters in particular. Another method that has been used frequently for the characterization of hydrogel foams is $\mu$-CT. ${ }^{19,24}$ In this technique, the attenuation of X-rays by the material is detected and a 3D picture is reconstructed. The method is suitable for both dry and swollen samples. Although this method is undoubtedly the most precise method for the characterization of both dried and swollen hydrogel foams, several drawbacks should be considered. Firstly, the acquisition can take several hours, which is not very efficient if multiple series of samples need to be characterised. Secondly, the acquisition time steeply increases with increasing resolution, which makes the characterization of the very thin pore opening boundaries even more timeconsuming. Thirdly, the equipment is extremely expensive and only few research facilities have access to such instruments. Alternatively, the pore diameters of swollen hydrogel foams can be examined by bright field microscopy as the outlines of the pores (Figure 6, middle) can be distinguished. However, due to the low contrast between the hydrogel matrix and the medium, detailed morphological structures such as pore openings are difficult to analyse. By contrast, confocal fluorescence microscopy enables the visualization of both pore and pore opening diameters in the swollen state due to the high contrast between the hydrogel matrix and the aqueous medium. Compared to what can be achieved by bright field microscopy, the interconnected structure can be visualised much better. As the picture acquisition time is only a matter of minutes, the method can be used to characterise multiple series of samples. However, there is a general limitation of confocal fluorescence microscopy when analysing porous materials. Due to the porous structure, some of the excitation light as well as the fluorescence emission are scattered when passing through the sample. Hence, the depth for picture acquisition is limited to approx. $220 \mu \mathrm{m}$ in the case of GM10 hydrogel foams. As a result, thicker samples cannot be analysed without cutting them. Therefore, the samples must be cut and visualised several times to ensure thorough characterization. Nevertheless, confocal fluorescence microscopy can be considered an alternative to $\mu$-CT for the visualization of swollen hydrogel foams when analysing the system described in this study. 


\subsection{Control of the pore opening diameter}

\subsubsection{Addition of polymer solution}

To decouple the bubble diameter from the gas fraction, the liquid content of the foam templates was varied by adding polymer solution to the generated foam at flow rate $v_{\mathrm{A}}$. The liquid foams were successfully cross-linked by irradiation with UV light to yield hydrogel foams. Crosslinked foams were swollen to equilibrium and analysed using confocal fluorescence microscopy. Pictures taken by confocal fluorescence microscopy are depicted in Figure 7 together with the flow rate $v_{\mathrm{A}}$ of the additionally added polymer solution. The obtained values for pore diameters and pore opening diameters, as well as their ratios and gas fractions are listed in Table 1. Other than in Section 3.2.2, the bubble diameter $\left(\left\langle D_{\mathrm{b}}\right\rangle=174 \pm 2 \mu \mathrm{m}\right)$ was only measured once, since the experimental parameters used for bubble formation were not changed.
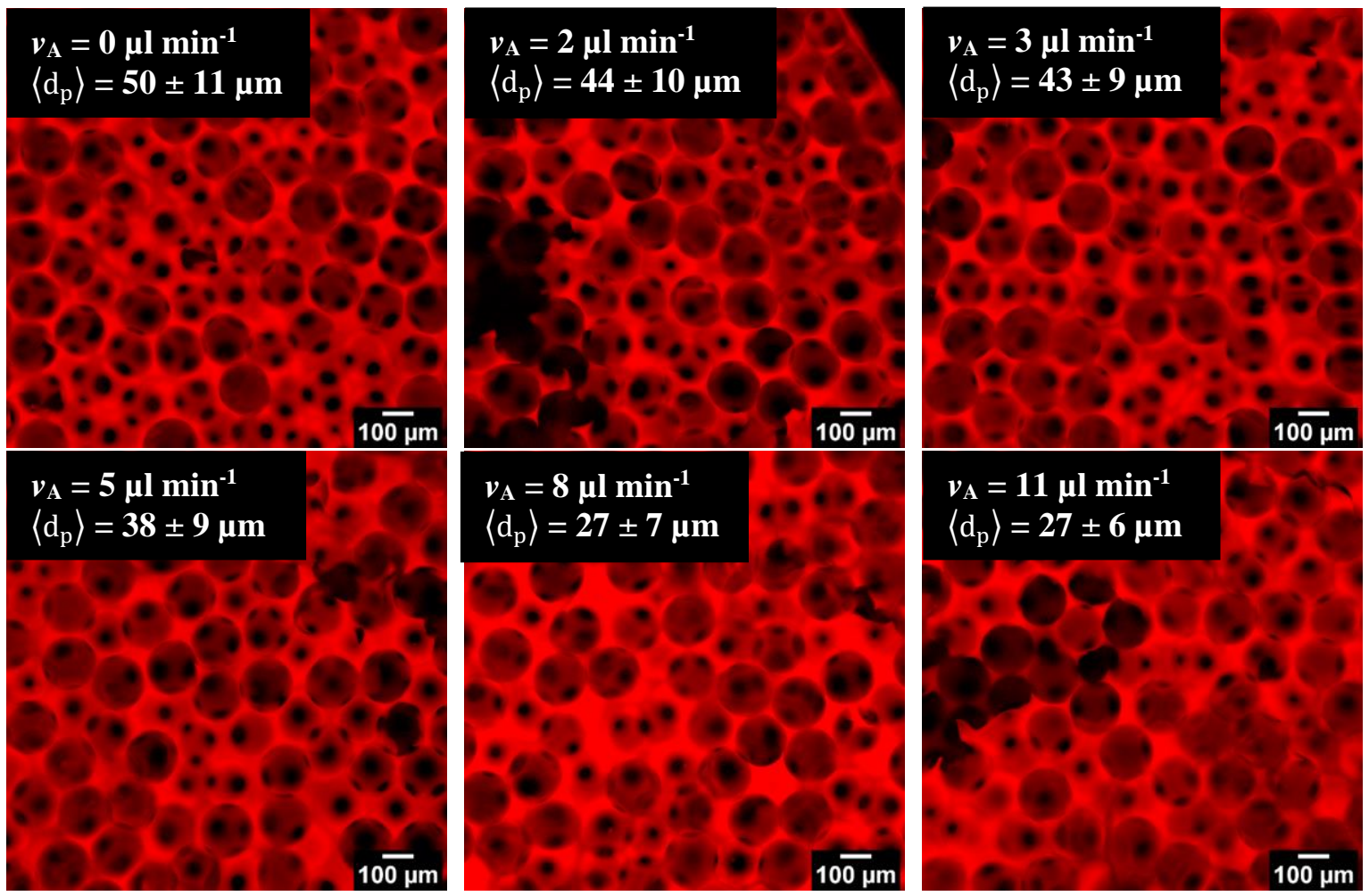

Figure 7: Pictures of GM10 foams taken with confocal fluorescence microscopy. The flow rate $v_{\mathrm{A}}$ and the average pore opening diameter $\left\langle d_{p}\right\rangle$ are displayed in the inlets. 
Table 1: Liquid flow rates $v_{\mathrm{A}}$ of additionally added GM solution, pore diameters $\left\langle D_{p}\right\rangle$, pore opening diameters $\left\langle d_{p}\right\rangle$, the ratio $\left\langle d_{p}\right\rangle /\left\langle D_{p}\right\rangle$, and the gas fraction of the liquid foams. The mean bubble diameter $\left\langle D_{b}\right\rangle$ of the generated liquid foam templates was $174 \pm 2 \mu \mathrm{m}$.

\begin{tabular}{|c|c|c|c|c|}
\hline$v_{\mathrm{A}} / \mu \mathrm{L} \min ^{-1}$ & $\left\langle D_{\mathrm{p}}\right\rangle / \mu \mathrm{m}$ & $\left\langle d_{\mathrm{p}}\right\rangle / \mu \mathrm{m}$ & $\left\langle d_{\mathrm{p}}\right\rangle /\left\langle D_{\mathrm{p}}\right\rangle$ & gas fraction /\% \\
\hline 0 & $178 \pm 4$ & $50 \pm 11$ & 0.28 & $82 \pm 4$ \\
\hline 2 & $175 \pm 4$ & $44 \pm 10$ & 0.25 & $80 \pm 1$ \\
\hline 3 & $181 \pm 5$ & $43 \pm 9$ & 0.24 & $79 \pm 1$ \\
\hline 5 & $179 \pm 3$ & $38 \pm 7$ & 0.21 & $74 \pm 1$ \\
\hline 8 & $178 \pm 3$ & $27 \pm 7$ & 0.15 & $67 \pm 1$ \\
\hline 11 & $177 \pm 4$ & $27 \pm 6$ & 0.15 & $66 \pm 0 *$ \\
\hline
\end{tabular}

*As the gas fraction of samples produced with this liquid flow rate could only be determined from two samples which did not differ in weight, the obtained standard deviation is zero.

Looking at Figure 7 and Table 1, one sees that the gas fraction decreased with increasing $v_{\mathrm{A}}$ from $82 \%$ to $66 \%$, i.e. we decoupled bubble size and gas fraction. The pore opening diameter varied from $50 \pm 11 \mu \mathrm{m}$ to $27 \pm 6 \mu \mathrm{m}$, respectively, while the pore diameter remained constant. Thus, the ratio of pore opening diameter and pore diameter could be adjusted between 0.15 and 0.28. Figure 7 also illustrates that the gas fraction influences the degree of pore ordering in the hydrogel foam. When no additional polymer solution is added to the sample (top, left), the pores are more ordered compared to samples where additional polymer solution is added (bottom, right). It was also found that the number of pore openings per pore decreases when polymer solution is added with high flow rates such as $8 \mu \mathrm{L} \mathrm{min}^{-1}$ and $11 \mu \mathrm{L} \mathrm{min}{ }^{-1}$. Note that adding polymer solution can only be used to tailor the pore opening diameter of foams with a high initial gas fraction and thus a large bubble size. We thus used a different method to tailor the pore opening diameters for foams with low initial gas fractions and thus smaller bubble sizes.

\subsubsection{Variation of the bubbling frequency}

To decouple the bubble diameter from the gas fraction, the bubbling frequency during microfluidic bubbling was varied by increasing gas pressure and liquid flow rate at a constant ratio. The resulting bubble diameters as well as their corresponding production parameters and 
the initial gas fraction are listed in Table 2. The resulting foams were successfully cross-linked by irradiation with UV-light and were swollen in water for confocal fluorescence microscopy. Pictures taken by confocal fluorescence microscopy are depicted in Figure 8 together with the liquid flow rate $v_{\mathrm{L}}$ of the polymer solution during the production. The obtained values for pore diameters and pore opening diameters, as well as their ratios and the gas fraction are also listed in Table 2. Other than in Section 3.2.1, the bubble diameter was measured for each set of samples, as the experimental parameters for bubble formation were different. Looking at Table 2 , one sees that we worked with a constant bubble diameter of $\sim 140 \mu \mathrm{m}$, while the gas fraction increases from top to bottom. In addition, Table 2 and Figure 8 illustrate that the pore opening diameter increases from $19 \mu \mathrm{m}$ to $32 \mu \mathrm{m}$ in the same direction, while the pore diameter remained constant. Thus, the ratio of pore opening diameter and pore diameter varied between 0.14 and 0.23. Similar to Section 3.2.1, foams with higher gas fractions are more ordered.

Table 2: Liquid flow rates $v_{\mathrm{L}}$ and gas pressures $p$ used to produce GM10 foams, bubble diameters $\left\langle D_{b}\right\rangle$, pore diameters $\left\langle D_{p}\right\rangle$, pore opening diameters $\left\langle d_{p}\right\rangle$, the ratio $\left\langle d_{p}\right\rangle /\left\langle D_{p}\right\rangle$, and the gas fraction of the liquid foams.

\begin{tabular}{|c|c|c|c|c|c|c|}
\hline $\begin{array}{c}v_{\mathrm{L}} / \\
\mu \mathrm{L} \mathrm{min} \text { m }^{-1}\end{array}$ & $\begin{array}{c}p / \\
\mathrm{mbar}\end{array}$ & $\left\langle D_{\mathrm{b}}\right\rangle / \mu \mathrm{m}$ & $\left\langle D_{\mathrm{p}}\right\rangle / \mu \mathrm{m}$ & $\left\langle d_{\mathrm{p}}\right\rangle / \mu \mathrm{m}$ & $\left\langle d_{\mathrm{p}}\right\rangle /\left\langle D_{\mathrm{p}}\right\rangle$ & $\begin{array}{c}\text { gas fraction } / \\
\%\end{array}$ \\
\hline 20 & 200 & $140 \pm 5$ & $131 \pm 6$ & $18 \pm 4$ & 0.14 & $60 \pm 4$ \\
\hline 30 & 300 & $135 \pm 3$ & $133 \pm 3$ & $19 \pm 5$ & 0.14 & $54 \pm 1$ \\
\hline 40 & 400 & $138 \pm 3$ & $136 \pm 3$ & $19 \pm 4$ & 0.14 & $65 \pm 1$ \\
\hline 50 & 500 & $145 \pm 4$ & $138 \pm 3$ & $20 \pm 5$ & 0.15 & $68 \pm 1$ \\
\hline 60 & 600 & $144 \pm 2$ & $140 \pm 3$ & $24 \pm 6$ & 0.17 & $71 \pm 1$ \\
\hline 70 & 700 & $144 \pm 3$ & $144 \pm 3$ & $30 \pm 7$ & 0.21 & $75 \pm 2$ \\
\hline 80 & 800 & $142 \pm 2$ & $142 \pm 4$ & $32 \pm 8$ & 0.23 & $76 \pm 1$ \\
\hline
\end{tabular}



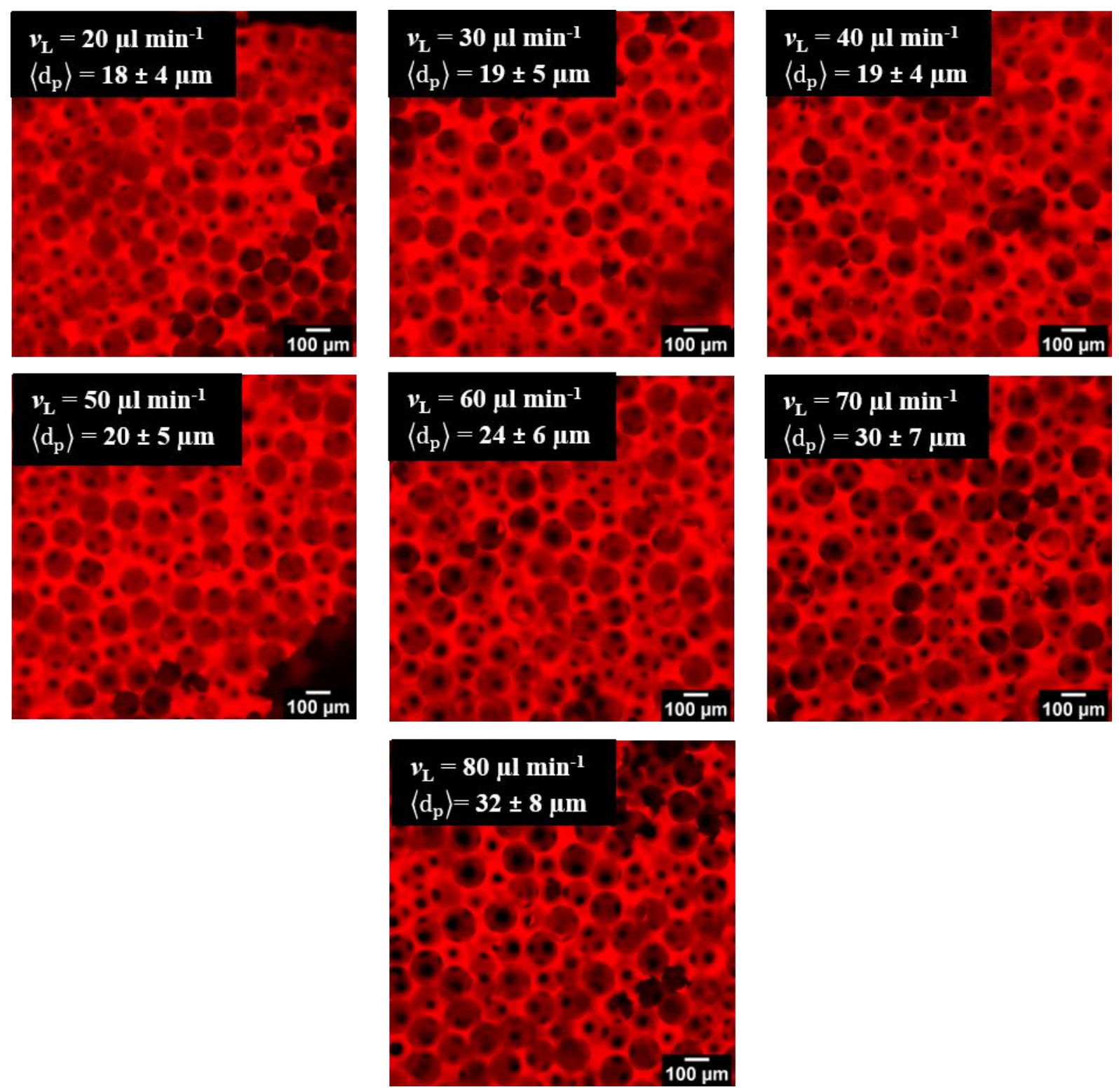

Figure 8: Pictures of GM10 foams taken with confocal fluorescence microscopy. The flow rate $v_{\mathrm{L}}$ and the average pore opening diameter $\left\langle d_{p}\right\rangle$ are displayed in the inlets. The ratio of flow rate $v_{\mathrm{L}}$ and gas pressure $p$ during foam generation was kept constant at $0.1 \mu \mathrm{L} \mathrm{min}^{-1} \mathrm{mbar}^{-1}$.

Please note that the gas fraction of foams produced with a liquid flow rate of $20 \mu \mathrm{L} \mathrm{min}{ }^{-1}$ or $30 \mu \mathrm{L} \mathrm{min}^{-1}$ is below $63.5 \%$, which corresponds to the critical packing limit for disordered foams. Theoretically, no pore openings should be observed for these samples. However, a drainage layer was observed at the bottom of the foams, which leads to an increased gas fraction in the foams and thus to the formation of pore openings. Consequently, the samples are not considered for further analysis in Section 3.3. For all other samples, no drainage layer was 
observed. To conclude one can say that the two techniques complement each other and allow generating hydrogel foams with controllable pore opening diameters at constant pore size.

\subsection{Comparison of pore opening diameters to bubble contact zones of liquid foams}

All experimentally obtained results for $\left\langle d_{\mathrm{p}}\right\rangle /\left\langle D_{\mathrm{p}}\right\rangle$ are plotted in Figure 9. Combining all results from Sections 3.2.1 and 3.2.2, one sees that $\left\langle d_{\mathrm{p}}\right\rangle /\left\langle D_{\mathrm{p}}\right\rangle$ can be varied between 0.14 and 0.28. The variation of $\left\langle d_{\mathrm{p}}\right\rangle /\left\langle D_{\mathrm{p}}\right\rangle$ was also examined for geopolymer foams by systematically adding the geopolymer-dispersion to a precursor foam generated from an aqueous surfactant solution. ${ }^{28,29}$ In these studies, the ratio of pore opening diameter to pore diameter was varied from 0.15 at low to 0.38 at very high gas fractions. Theoretically such high gas fractions could also be obtained for our system. However, since our gelatin-based hydrogel foams are crosslinked via radical polymerization, we encounter the more oxygen inhibition the higher the gas fraction is. Thus, a gas fraction of $82 \%$ represents the upper limit of the accessible pore opening diameters when generating foams under the conditions described in Section 2.3. By using a more rigorous inert gas atmosphere during foaming and cross-linking, larger gas fractions and thus also larger pore openings might be accessible. The lower limit of the pore opening diameters corresponds to a gas fraction of $65 \%$. Even though liquid foams with gas fractions of less than $65 \%$ can be generated, these foams instantaneously start to drain. As this competes with the rapid cross-linking of the polymer chains, inhomogeneous foams are generated which cannot be reliably compared to foams with gas fractions above $65 \%$. In a study by Costantini et al. ${ }^{18},\left\langle d_{\mathrm{p}}\right\rangle /\left\langle D_{\mathrm{p}}\right\rangle$ of alginate foams were varied by using surfactant concentrations between 0.6 wt. $\%$ and 5 wt. $\%$. The ratio of pore opening diameter to pore diameter varied between 0.25 and 0.32 . However, our techniques avoid the preparation and formulation of multiple solutions. Moreover, we can work at low surfactant concentrations which is of particular importance in applications such as tissue engineering. 
To a first approximation, the pore opening diameters $\left\langle d_{\mathrm{p}}\right\rangle$ are believed to be closely linked to the diameter of the contact zone $\left\langle d_{\mathrm{b}}\right\rangle$ between touching bubbles in the liquid template. The ratio of this contact zone $\left\langle d_{\mathrm{b}}\right\rangle$ and the bubble diameter $\left\langle D_{\mathrm{b}}\right\rangle$ can be estimated based on the gas fraction $\phi$ of the liquid foam. A prediction of this relation was derived by Princen for polydisperse disordered foams ${ }^{32}$ and adapted to monodisperse disordered systems by Arditty ${ }^{33}$

$$
\frac{\left\langle d_{\mathrm{b}}\right\rangle}{\left\langle D_{\mathrm{b}}\right\rangle}=\frac{0.6}{\phi^{\frac{1}{3}}}\left(1-\frac{1.74}{\left(\frac{\phi}{1-\phi}+1.3\right)^{\frac{1}{2}}}\right)^{\frac{1}{2}}
$$

To relate the gas fraction of the solid foam to that of the liquid template, we make the following assumption: As the bubble diameters of the liquid templates are almost equal to the pore diameters of the obtained hydrogel foams (see Tables 1 and 2), we assume that shrinkage during cross-linking and freeze-drying is counterbalanced by an isotropic swelling in water. Thus, the gas fraction in the hydrogel foam can be assumed to be equal to the gas fraction of the liquid template. The gas fractions of the liquid templates are listed in Tables 1 and 2. In Figure 9 we also plot the predictions of equation (3) for disordered foams and those obtained from Surface Evolver simulations by Pitois et al. ${ }^{28,34}$ for ordered foams (hexagonally close-packed - FCC). We can see that globally our data lies between the predictions for disordered and ordered foams, with a tendency to move from the disordered to the ordered case with increasing gas fraction. This tendency is confirmed by visual inspection of the samples, which, as discussed in Sections 3.2.1 and 3.2.2, show increased local ordering of the bubbles with increasing gas fraction. This can be explained by the fact that bubble interactions become stronger for increasing gas fraction, which, in turn, increases the driving force towards an energetically more favourable packing. For monodisperse foams, this packing is the FCC structure. 


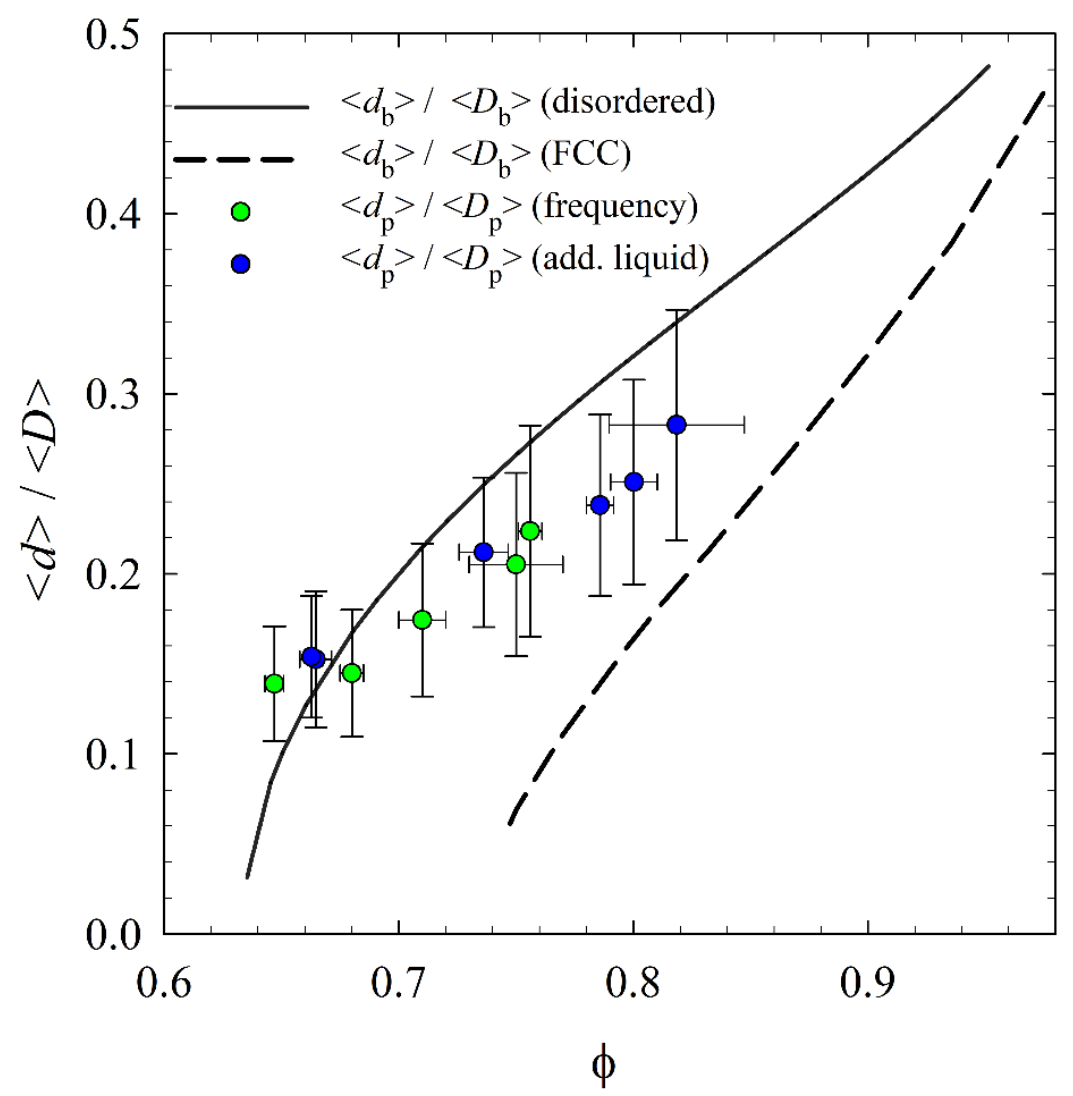

Figure 9: Ratio of experimentally determined pore opening diameters $\left\langle d_{\mathrm{p}}\right\rangle$ and pore diameters $\left\langle D_{\mathrm{p}}\right\rangle$ obtained by adding liquid (blue data) and by varying the bubbling frequency (green data) plotted against the gas fraction $\phi$ of the foam. Also plotted are theoretical predictions calculated from equation (3) for disordered foams (solid line) or obtained by Surface Evolver simulations for ordered foams (dotted line $)^{34}$. The errors are determined from an error propagation.

\section{Conclusions}

We describe two approaches to the independent variation of the pore diameter and the pore opening diameter in hydrogel foams generated by microfluidic bubbling. The pore opening diameter was decoupled from the pore diameter by varying the gas fraction of the templating foam at constant bubble size. To this end, two complementary methods were used. The liquid fraction was varied (1) by adding polymer solution to the generated liquid foams and (2) by changing the bubbling frequency. The pore openings were visualised with confocal fluorescence microscopy, which allows for a fast characterisation of the hydrogel foams in their equilibrium swollen state. We successfully varied the ratio of pore opening diameters to pore 
diameters $\left\langle d_{\mathrm{p}}\right\rangle /\left\langle D_{\mathrm{p}}\right\rangle$ between 0.14 and 0.28 . As the pore openings are hypothesised to be generated by the rupture of thin films that form between neighbouring bubbles touching each other, the area of the thin films can be predicted based on surface area minimisations of bubble assemblies. ${ }^{32,33}$ We show that our data lies in between the theoretical predictions obtained for disordered foams and Surface Evolver simulations for ordered (FCC) foams ${ }^{34}$. Our $\left\langle d_{\mathrm{p}}\right\rangle /\left\langle D_{\mathrm{p}}\right\rangle$ ratios correlate well with the predicted values for disordered foams at low gas fractions, indicating that the pore opening sizes are indeed determined by the thin film sizes in the template. For higher gas fractions, the ratios $\left\langle d_{\mathrm{p}}\right\rangle /\left\langle D_{\mathrm{p}}\right\rangle$ approach predicted values for FCC ordered foams. In other words, we observe an increasing degree of ordering for hydrogel foams with increasing gas fraction. Despite being of great importance in the field of biomaterials, the variation of the pore opening in chemically cross-linked polymer foams was examined in very few other studies. Costantini et al. ${ }^{18}$ used different surfactant concentrations between $0.6 \mathrm{wt} . \%$ and 5 wt. $\%$ to vary the thin film area, while Testouri et $a l .{ }^{27}$ qualitatively examined the pore opening diameter in free standing foams subjected to drainage. The variation of the pore opening in geopolymer foams has been achieved by letting generated precursor foams drain and adding a cross-linking geopolymer dispersion afterwards. ${ }^{28,29}$ However, these approaches are either time-consuming or involve the formulation of multiple solutions. With the methods presented here, it is possible to systematically vary the influence of the gas fraction of the foam template on the pore opening size while working at low surfactant concentrations. By decoupling the bubble diameter from the gas fraction and thus the pore opening diameter from the pore diameter, it is now possible to independently study the influence of pore size and pore opening size on the behaviour of cells in tissue engineering, which is the subject of ongoing research. In addition to that, a new arena in the field of adsorption and release has opened up as the staining of the hydrogel foams for confocal microscopy relies on a cation exchange with 
methylene blue. This effect could be used for drug delivery or wastewater treatment where cationic drugs or pollutants are adsorbed on the hydrogel matrix.

\section{Acknowledgements}

Friederike Dehli wants to thank Fabian Moczko for fruitful discussions. We thank Dr. Marco Costantini for the fabrication of the polycarbonate chip at the Polish Academy of Sciences, Warsaw. Friederike Dehli gratefully acknowledges financial support by the Fonds der Chemischen Industrie. The authors gratefully acknowledge the Technology Platform "Cellular Analytics“ of the Stuttgart Research Center Systems Biology for their support and assistance in this work. Part of this work has been conducted within the IdEx Unistra framework (Chair W. Drenckhan) and has, as such, benefited from funding from the state, managed by the French National Research Agency as part of the "Investments for the future" program. The work was also supported by an ERC consolidator grant (agreement 819511-METAFOAM). Cosima Stubenrauch acknowledges a fellowship by the Institute of Advanced Studies at the University of Strasbourg (USIAS).

\section{References}

(1) Ahmed, E. M. Hydrogel: Preparation, Characterization, and Applications: A Review. J. Adv. Res. 2015, 6 (2), 105-121. https://doi.org/10.1016/j.jare.2013.07.006.

(2) Peppas, B. N. A.; Hilt, J. Z.; Khademhosseini, A.; Langer, R. Hydrogels in Biology and Medicine : From Molecular Principles to Bionanotechnology. Adv. Mater. 2006, 18, 1345-1360. https://doi.org/10.1002/adma.200501612.

(3) Zhu, J.; Marchant, R. E. Design Properties of Hydrogel Tissue-Engineering Scaffolds. Expert Rev. Med. Devices 2011, 8 (5), 607-626. https://doi.org/10.1586/erd.11.27.

(4) Lai, J. Y.; Li, Y. T. Functional Assessment of Cross-Linked Porous Gelatin Hydrogels for Bioengineered Cell Sheet Carriers. Biomacromolecules 2010, 11 (5), 1387-1397. https://doi.org/10.1021/bm100213f.

(5) Miyamoto, K.; Sasaki, M.; Minamisawa, Y.; Kurahashi, Y.; Kano, H.; Ishikawa, S. I. Evaluation of in Vivo Biocompatibility and Biodegradation of Photocrosslinked Hyaluronate Hydrogels (HADgels). J. Biomed. Mater. Res. - Part A 2004, 70 (4), 550559. https://doi.org/10.1002/jbm.a.30112.

(6) Ueng, S. W. N.; Yuan, L. J.; Lee, N.; Lin, S. S.; Chan, E. C.; Weng, J. H. In Vivo Study of Biodegradable Alginate Antibiotic Beads in Rabbits. J. Orthop. Res. 2004, 22 (3), 592-599. https://doi.org/10.1016/j.orthres.2003.09.001.

(7) Luo, Y.; Kirker, K. R.; Prestwich, G. D. Cross-Linked Hyaluronic Acid Hydrogel Films New Biomaterials. J. Control. Release 2000, 69, 169-184.

(8) Augst, A. D.; Kong, H. J.; Mooney, D. J. Alginate Hydrogels as Biomaterials. Macromol. Biosci. 2006, 6 (8), 623-633. https://doi.org/10.1002/mabi.200600069.

(9) Dollinger, C.; Ciftci, S.; Knopf-Marques, H.; Guner, R.; Ghaemmaghami, A. M.; 
Debry, C.; Barthes, J.; Vrana, N. E. Incorporation of Resident Macrophages in Engineered Tissues: Multiple Cell Type Response to Microenvironment Controlled Macrophage-Laden Gelatine Hydrogels. J. Tissue Eng. Regen. Med. 2018, 12 (2), 330340. https://doi.org/10.1002/term.2458.

(10) Barbetta, A.; Massimi, M.; Di Rosario, B.; Nardecchia, S.; De Colli, M.; Devirgiliis, L. C.; Dentini, M. Emulsion Templated Scaffolds That Include Gelatin and Glycosaminoglycans. Biomacromolecules 2008, 9 (10), 2844-2856. https://doi.org/10.1021/bm800599d.

(11) Nichol, J. W.; Koshy, S. T.; Bae, H.; Hwang, C. M.; Yamanlar, S.; Khademhosseini, A. Cell-Laden Microengineered Gelatin Methacrylate Hydrogels. Biomaterials 2010, 31 (21), 5536-5544. https://doi.org/10.1016/j.biomaterials.2010.03.064.

(12) Ratnikov, B.; Deryugina, E.; Leng, J.; Marchenko, G.; Dembrow, D.; Strongin, A. Determination of Matrix Metalloproteinase Activity Using Biotinylated Gelatin. Anal. Biochem. 2000, 286 (1), 149-155. https://doi.org/10.1006/abio.2000.4798.

(13) Majer, G.; Southan, A. Adenosine Triphosphate Diffusion through Poly(Ethylene Glycol) Diacrylate Hydrogels Can Be Tuned by Cross-Link Density as Measured by PFG-NMR. J. Chem. Phys. 2017, 146 (22), 225101. https://doi.org/10.1063/1.4984979.

(14) Lieleg, O.; Ribbeck, K. Biological Hydrogels as Selective Diffusion Barriers. Trends Cell Biol. 2011, 21 (9), 543-551. https://doi.org/10.1016/j.tcb.2011.06.002.

(15) Peppas, N. A.; Lustig, S. R. And Relaxations of the Macromolecular Carrier in the Diffusional Release of Conceptual and Scaling Relationships. Ann. New York Acad. Sci. 1985, 446, 26-41.

(16) Andrieux, S.; Drenckhan, W.; Stubenrauch, C. Highly Ordered Biobased Scaffolds: From Liquid to Solid Foams. Porous Polymers: A Special Issue of Polymer 2017, 216, 425-431. https://doi.org/10.1016/j.polymer.2017.04.031.

(17) Barbetta, A.; Rizzitelli, G.; Bedini, R.; Pecci, R.; Dentini, M. Porous Gelatin Hydrogels by Gas-in-Liquid Foam Templating. Soft Matter 2010, 6 (8), 1785-1792. https://doi.org/10.1039/b920049e.

(18) Costantini, M.; Colosi, C.; Jaroszewicz, J.; Tosato, A.; Wojciech, S.; Dentini, M.; Garstecki, P.; Barbetta, A. Microfluidic Foaming : A Powerful Tool for Tailoring the Morphological and Permeability Properties of Sponge-like Biopolymeric Scaffolds. ACS Appl. Mater. Interfaces 2015, 7, 23660-23671.

https://doi.org/10.1021/acsami.5b08221.

(19) Colosi, C.; Costantini, M.; Barbetta, A.; Pecci, R.; Bedini, R.; Dentini, M. Morphological Comparison of PVA Scaffolds Obtained by Gas Foaming and Microfluidic Foaming Techniques. Langmuir 2013, 29 (1), 82-91. https://doi.org/10.1021/la303788z.

(20) Langlois, V.; Trinh, V. H.; Lusso, C.; Perrot, C.; Chateau, X.; Khidas, Y.; Pitois, O. Permeability of Solid Foam: Effect of Pore Connections. Phys. Rev. E 2018, 97 (5), 110. https://doi.org/10.1103/PhysRevE.97.053111.

(21) Trinh, V. H.; Langlois, V.; Guilleminot, J.; Perrot, C.; Khidas, Y.; Pitois, O. Tuning Membrane Content of Sound Absorbing Cellular Foams: Fabrication, Experimental Evidence and Multiscale Numerical Simulations. Mater. Des. 2019, 162, 345-361. https://doi.org/10.1016/j.matdes.2018.11.023.

(22) Madden, L. R.; Mortisen, D. J.; Sussman, E. M.; Dupras, S. K.; Fugate, J. A.; Cuy, J. L.; Hauch, K. D.; Laflamme, M. A.; Murry, C. E.; Ratner, B. D. Proangiogenic Scaffolds as Functional Templates for Cardiac Tissue Engineering. Proc. Natl. Acad. Sci. U. S. A. 2010, 107 (34), 15211-15216. https://doi.org/10.1073/pnas.1006442107.

(23) Lutzweiler, G.; Barthes, J.; Koenig, G.; Kerdjoudj, H.; Mayingi, J.; Boulmedais, F.; Schaaf, P.; Drenckhan, W.; Vrana, N. E. Modulation of Cellular Colonization of Porous Polyurethane Scaffolds via the Control of Pore Interconnection Size and 
Nanoscale Surface Modifications. ACS Appl. Mater. Interfaces 2019, 11 (22), 1981919829. https://doi.org/10.1021/acsami.9b04625.

(24) Costantini, M.; Colosi, C.; Mozetic, P.; Jaroszewicz, J.; Tosato, A.; Rainer, A.; Trombetta, M.; Wojciech, Ś.; Dentini, M.; Barbetta, A. Correlation between Porous Texture and Cell Seeding Efficiency of Gas Foaming and Microfluidic Foaming Scaffolds. Mater. Sci. Eng. C 2016, 62, 668-677. https://doi.org/10.1016/j.msec.2016.02.010.

(25) Oh, S. H.; Kim, T. H.; Im, G. Il; Lee, J. H. Investigation of Pore Size Effect on Chondrogenic Differentiation of Adipose Stem Cells Using a Pore Size Gradient Scaffold. Biomacromolecules 2010, 11 (8), 1948-1955. https://doi.org/10.1021/bm100199m.

(26) Dehli, F.; Rebers, L.; Stubenrauch, C.; Southan, A. Highly Ordered Gelatin Methacryloyl Hydrogel Foams with Tunable Pore Size. Biomacromolecules 2019, 20 (7), 2666-2674. https://doi.org/10.1021/acs.biomac.9b00433.

(27) Testouri, A.; Ranft, M.; Honorez, C.; Kaabeche, N.; Ferbitz, J.; Freidank, D.; Drenckhan, W. Generation of Crystalline Polyurethane Foams Using Millifluidic Labon-a-Chip Technologies. Adv. Eng. Mater. 2013, 15 (11), 1086-1098. https://doi.org/10.1002/adem.201300077.

(28) Pitois, O.; Kaddami, A.; Langlois, V. Permeability of Monodisperse Solid Foams. Transp. Porous Media 2020, 134 (3), 635-649. https://doi.org/10.1007/s11242-02001461-6.

(29) Langlois, V.; Kaddami, A.; Pitois, O.; Perrot, C. Acoustics of Monodisperse Open-Cell Foam: An Experimental and Numerical Parametric Study. J. Acoust. Soc. Am. 2020, 148 (3), 1767-1778. https://doi.org/10.1121/10.0001995.

(30) Costantini, M.; Colosi, C.; Guzowski, J.; Barbetta, A.; Jaroszewicz, J.; Swieszkowski, W.; Dentini, M.; Garstecki, P. Highly Ordered and Tunable PolyHIPEs by Using Microfluidics. J. Mater. Chem. B 2014, 2, 2290-2300. https://doi.org/10.1039/c3tb21227k.

(31) Garstecki, P.; Gitlin, I.; Diluzio, W.; Whitesides, G. M.; Kumacheva, E.; Stone, H. A. Formation of Monodisperse Bubbles in a Microfluidic Flow-Focusing Device. Appl. Phys. Lett. 2004, 85 (13), 2649-2651. https://doi.org/10.1063/1.1796526.

(32) Princen, H. M. Pressure/Volume/Surface Area Relationships in Foams and Highly Concentrated Emulsions: Role of Volume Fraction. Langmuir 1988, 4 (1), 164-169. https://doi.org/10.1021/la00079a030.

(33) Arditty, S. Fabrication, Stability and Rheological Properties of Particles-Stabilized Emulsions, PhD Thesis, University of Bordeaux (2004).

(34) Pitois, O.; Kaddami, A.; Langlois, V. Capillary Imbibition in Open-Cell Monodisperse Foams. J. Colloid Interface Sci. 2020, 571, 166-173. https://doi.org/10.1016/j.jcis.2020.03.013.

(35) Claaßen, C.; Claaßen, M. H.; Truffault, V.; Sewald, L.; Tovar, G. E. M.; Borchers, K.; Southan, A. Quantification of Substitution of Gelatin Methacryloyl: Best Practice and Current Pitfalls. Biomacromolecules 2018, 19 (1), 42-52. https://doi.org/10.1021/acs.biomac.7b01221.

(36) Van Den Bulcke, A. I.; Bogdanov, B.; De Rooze, N.; Schacht, E. H.; Cornelissen, M.; Berghmans, H. Structural and Rheological Properties of Methacrylamide Modified Gelatin Hydrogels. Biomacromolecules 2000, 1 (1), 31-38. https://doi.org/10.1021/bm990017d.

(37) Andrieux, S.; Drenckhan, W.; Stubenrauch, C. Generation of Solid Foams with Controlled Polydispersity Using Microfluidics. Langmuir 2018, 34 (4), 1581-1590. https://doi.org/10.1021/acs.langmuir.7b03602.

(38) Sewald, L.; Claaßen, C.; Götz, T.; Claaßen, M. H.; Truffault, V.; Tovar, G. E. M.; 
Southan, A.; Borchers, K. Beyond the Modification Degree: Impact of Raw Material on Physicochemical Properties of Gelatin Type A and Type B Methacryloyls.

Macromol. Biosci. 2018, 18 (12), 1-10. https://doi.org/10.1002/mabi.201800168.

(39) Zhang, G.; Shuang, S.; Dong, C.; Pan, J. Study on the Interaction of Methylene Blue with Cyclodextrin Derivatives by Absorption and Fluorescence Spectroscopy. Spectrochim. Acta Part A 2003, 59, 2935-2941. https://doi.org/10.1016/S13861425(03)00123-9.

(40) Barbetta, A.; Carrino, A.; Costantini, M.; Dentini, M. Polysaccharide Based Scaffolds Obtained by Freezing the External Phase of Gas-in-Liquid Foams. Soft Matter 2010, 6 (20), 5213-5224. https://doi.org/10.1039/c0sm00616e.

(41) Meagher, A. J.; Whyte, D.; Banhart, J.; Hutzler, S.; Weaire, D.; García-Moreno, F. Slow Crystallisation of a Monodisperse Foam Stabilised against Coarsening. Soft Matter 2015, 11 (23), 4710-4716. https://doi.org/10.1039/c4sm02412e. 


\section{SUPPORTING INFORMATION}

Tailoring and visualising pore openings in gelatin-based hydrogel foams

Friederike Dehli ${ }^{1}$, Alexander Southan ${ }^{2}$, Wiebke Drenckhan ${ }^{3}$, Cosima Stubenrauch $^{1,4^{*}}$

${ }^{1}$ Institute of Physical Chemistry, University of Stuttgart, Pfaffenwaldring 55, 70569 Stuttgart, Germany

${ }^{2}$ Institute of Interfacial Process Engineering and Plasma Technology, University of Stuttgart, Nobelstraße 12, 70569 Stuttgart, Germany

${ }^{3}$ Institut Charles Sadron, 23 rue de Loess, Strasbourg, 67034, France

${ }^{4}$ Institute of Advanced Studies (USIAS), University of Strasbourg, F-67000 France

*Corresponding Author : cosima.stubenrauch@ipc.uni-stuttgart.de, 0049711 685-64470 


\section{Calibration of microfluidic chip}

The range of accessible bubble sizes was obtained by varying the liquid flow rate or the gas pressure. Figure S1 depicts the obtained bubble sizes as a function of the gas pressure and the liquid flow rate, respectively.
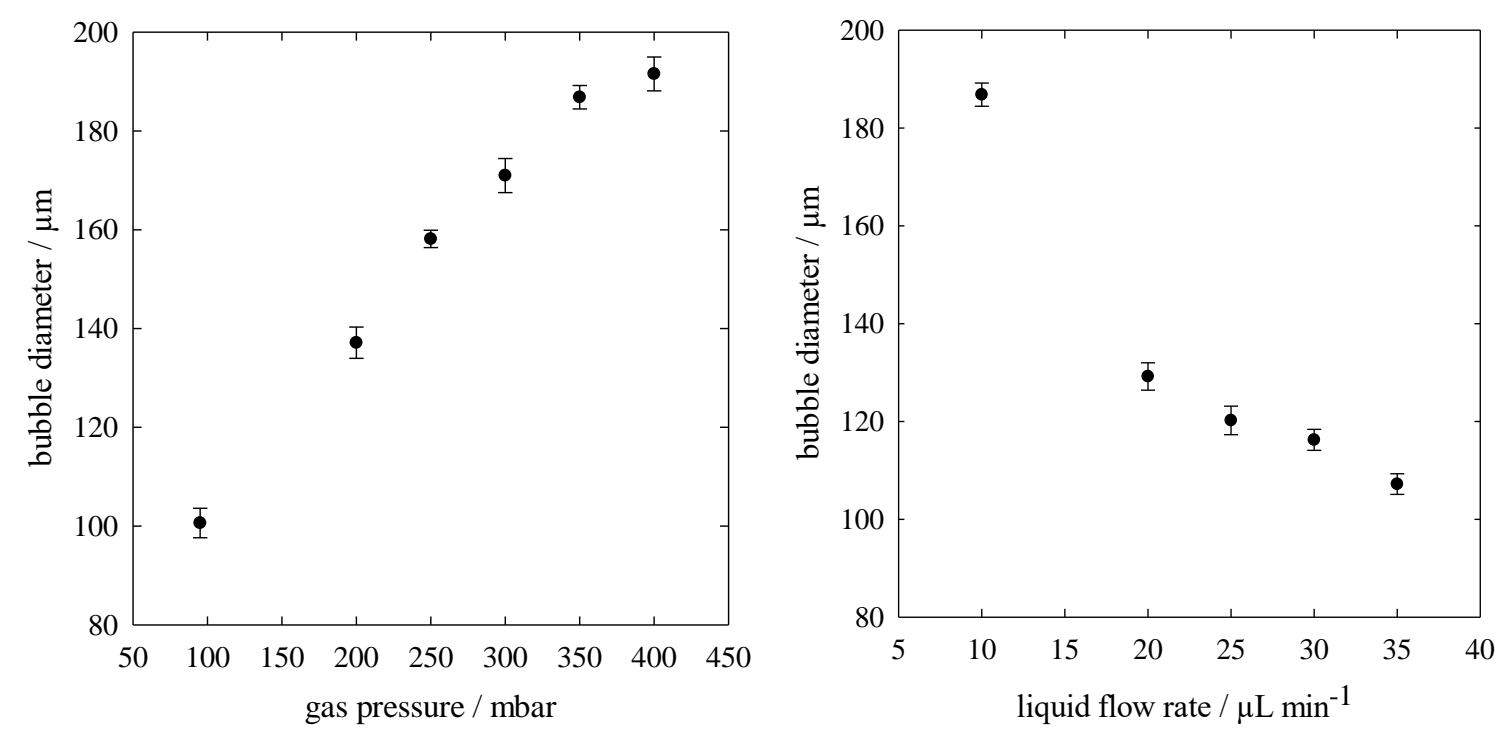

Figure S1: Bubble sizes of liquid foams as a function of (left) the gas pressure at a liquid flow rate of $10 \mu \mathrm{L} \mathrm{min}^{-1}$ and (right) the liquid flow rate at a gas pressure of $350 \mathrm{mbar}$.

\section{Surface areas of samples}

The surfaces of the samples display an ordered morphology - the bubbles crystallise at the sample mold walls and the quartz glass plate. Exemplary images of the first bubble layer for different samples are depicted in Figure S2.
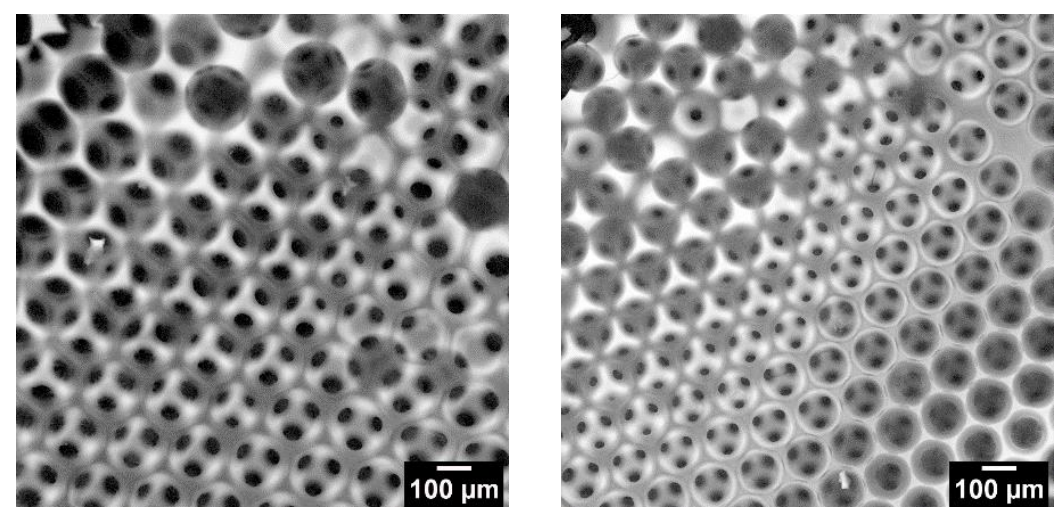

Figure S2: Pictures of surface layers for different samples: (left) The sample was prepared according to the method described in Section 2.3.1. Additional liquid was added with a flow rate of $3 \mu \mathrm{L} \mathrm{min}^{-1}$. (right) The sample was prepared according to the method described in Section 2.3.2. A liquid flow rate of $50 \mu \mathrm{L} \mathrm{min}^{-1}$ and a gas pressure of $500 \mathrm{mbar}$ was used. 


\section{Distributions of pore diameters and pore opening diameters}

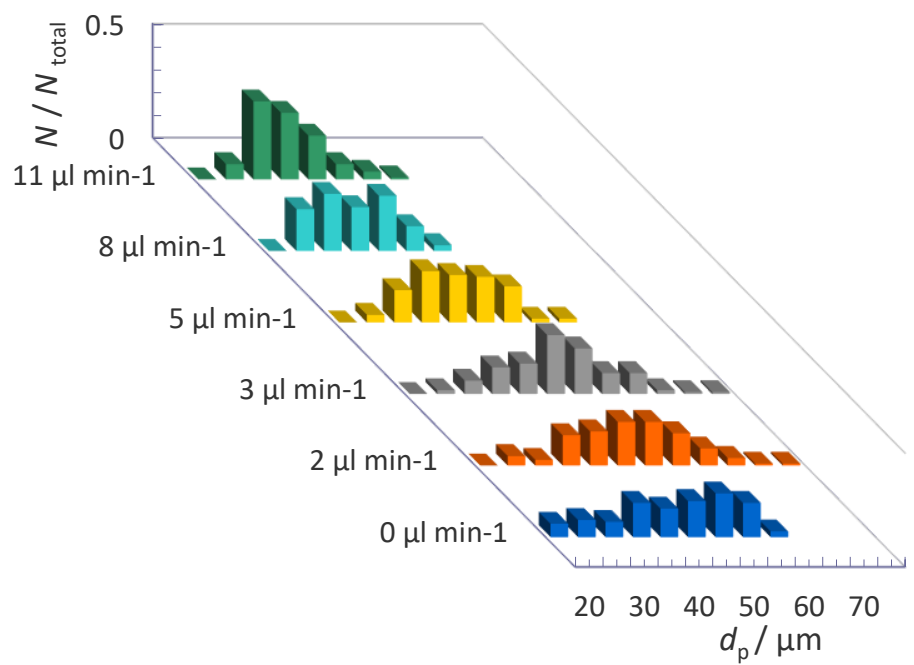

Figure S3: Distribution of pore opening diameters when additional polymer solution was added to the liquid templates (see Sections 2.3.1 and 3.2.1).

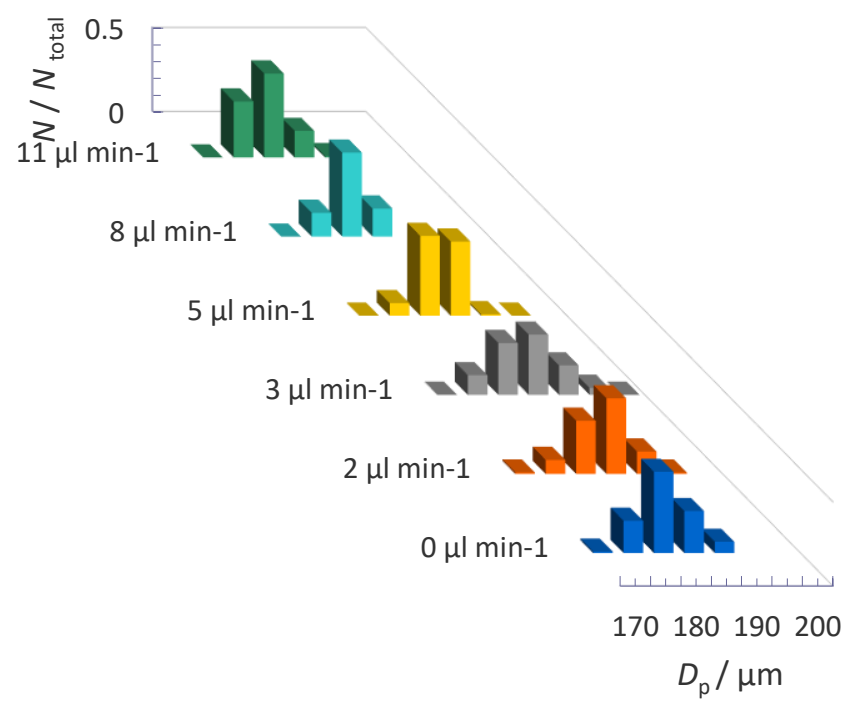

Figure S4: Distribution of pore diameters when additional polymer solution was added to the liquid templates (see Sections 2.3.1 and 3.2.1). 


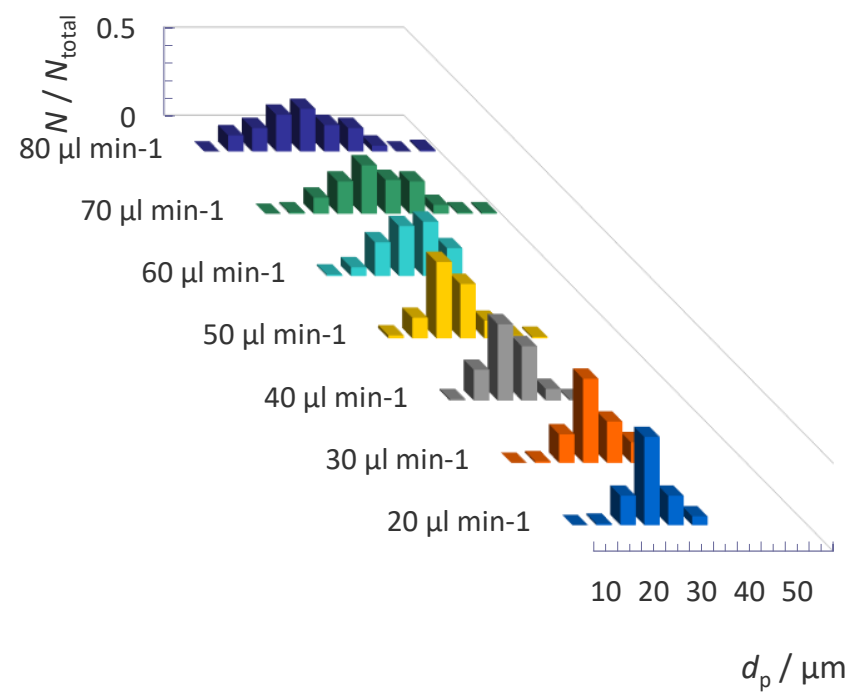

Figure S5: Distribution of pore opening diameters for samples produced with different bubbling frequencies (see Sections 2.3.2 and 3.2.2).

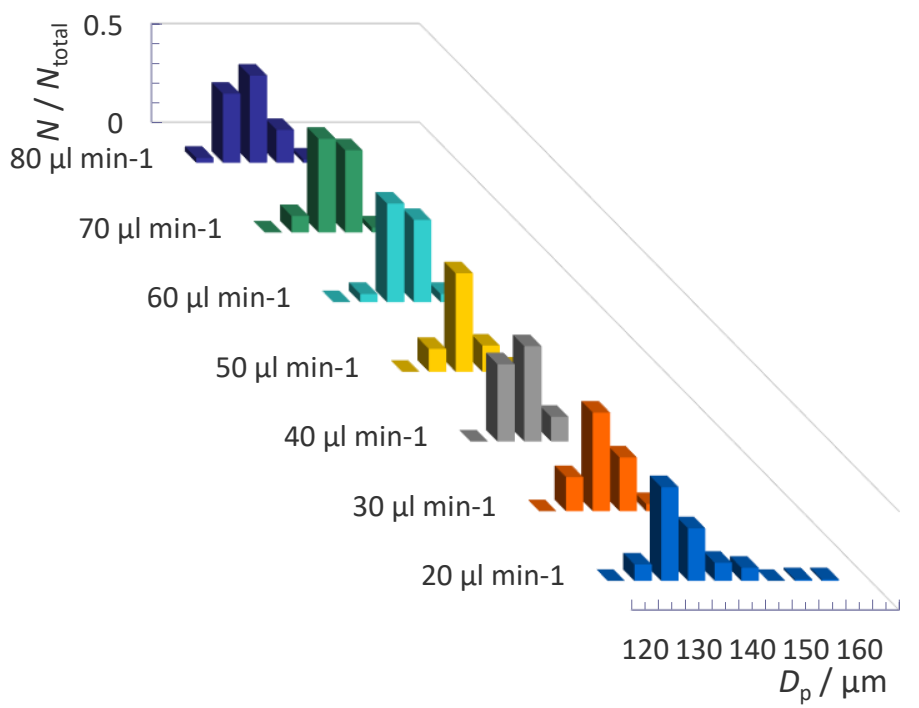

Figure S6: Distribution of pore diameters for samples produced with different bubbling frequencies (see Sections 2.3.2 and 3.2.2). 